

\title{
Physical Simulation of Land Vehicles with Obstacle Avoidance and Various Terrain Interactions
}

\author{
Stéphane Jimenez, Annie Luciani, Olivier Raoult
}

\section{To cite this version:}

Stéphane Jimenez, Annie Luciani, Olivier Raoult. Physical Simulation of Land Vehicles with Obstacle Avoidance and Various Terrain Interactions. Journal of Visualization and Computer Animation, 1993, 4 (2), pp.79-94. 10.1002/vis.4340040204 . hal-00486127

\section{HAL Id: hal-00486127 https://hal.science/hal-00486127}

Submitted on 22 Apr 2014

HAL is a multi-disciplinary open access archive for the deposit and dissemination of scientific research documents, whether they are published or not. The documents may come from teaching and research institutions in France or abroad, or from public or private research centers.
L'archive ouverte pluridisciplinaire HAL, est destinée au dépôt et à la diffusion de documents scientifiques de niveau recherche, publiés ou non, émanant des établissements d'enseignement et de recherche français ou étrangers, des laboratoires publics ou privés. 


\title{
Physical Simulation of Land Vehicles with Obstacle Avoidance and Various Terrain Interactions
}

\author{
STÉPHANE JIMENEZ, ANNIE LUCIANI AND OLIVIER RAOULT \\ ACROE-IMAG, Association pour la Création et la Recherche sur les Outils \\ d'Expression, Institut d'Informatique et de Mathématiques Appliquées de Grenoble, 46 \\ av. Félix Viallet, 38000 Grenoble, France
}

\begin{abstract}
SUMMARY
Programming the motions of an autonomous planetary robot moving in an hostile and hazardous environment is a complex task which requires both the construction of nominal motion plans and the anticipation as far as possible of the effects of the interactions existing between the vehicle and the terrain. In this paper we show how physical models and dynamic simulation tools can be used for amending and completing a nominal motion plan provided by a classical geometrical path planner. The purpose of our physical modeller-simulator is to anticipate the dynamic behaviour of the vehicle while executing the nominal motion plan. Then the obtained simulation results can be used to assess and optimize the nominal motion plan. In the first part, we outline the physical models that have been used for modelling the different types of vehicle, of terrain and of vehicle-surface interactions. Then we formulate the motion planning problem through the definition of two basic abstract constructions derived from physical model: the concept of generalized obstacle and the concept of physical target. We show with various examples how it is possible, when using this method, to solve the locomotion problem and the obstacle avoidance problem simultaneously and, furthermore, to provide the human operator with a true force feedback gestural control over the simulated robot.
\end{abstract}

KEY WORDS Motion control Physically based modelling Driving simulator Real-time animation and simulation

\section{INTRODUCTION}

The work presented here is a contribution to a robotic project which involves a complete system for the teleprogramming of an autonomous planetary rover. We are mainly concerned in this system with the optimization and the assessment of robust trajectories for the autonomous rover. More generally the problem is how to plan the motions for a complex articulated land vehicle moving in a natural environment. Geometrical and dynamical aspects will have to be considered in this problem.

During the ten past years, robotics researchers have mainly focused on the problem of planning the motions for a robot. Most of the research has dealt with path planning, i.e. the problem of finding collision-free trajectories between two given configurations of the robot.

Because of the intrinsic complexity of motion planning, many researchers have

$1049-8907 / 93 / 020079-16 \$ 13.00$

Received June 1992

(C) 1993 by John Wiley \& Sons, Ltd. 
solved a subset of the problem using purely geometrical approaches. Despite a great ability to solve geometrical problems, the effective generation of viable paths for a robot having numerous degrees of freedom and moving in a natural environment is beyond the capabilities of existing geometrical reasoning based implementations.

More recent results have been obtained in the field of motion planning. They involve planning methods dealing with non-integrable kinematic constraints such as non-holonomic constraints. ${ }^{1,2}$ Other methods deal with trajectory optimizations under kinematic constraints. ${ }^{3}$ These methods cannot be efficiently applied in our motion planning problem, which concerns a complex robot moving in a natural and little known environment: in this situation, the physical interactions existing between the vehicle and the ground are of prime importance, because frictions, slidings, skids and grip phenomena can influence the robot motion.

The work we have contributed to is based on a new method for solving motion planning problems in the case of a natural environment containing both obstacles that can be crossed over by the vehicle and obstacles that have to be avoided.

The method involves adapted tools to model and to simulate the dynamic aspects of the problem. A physical simulation of the task performance provides an additional prediction tool of the robot's behaviour.

The use of physical models means that the geometrical models of the ground and of the vehicle must first be converted into appropriate representations. It also led us to combine the physical simulation and the motion planning capabilities through two complementary concepts: the concept of 'generalized obstacles' and the concept of 'physical target'.

Beyond the motion planning application, these concepts have led us towards a pertinent means of controlling the motions in a dynamic simulation.

\section{TOOLS FOR PHYSICAL MODELLING AND SIMULATION}

\section{The physical modeller-simulator Cordis-Anima}

The physical model does not describe movement, but what generates it, i.e. the physical object itself. Thus, with a physical representation, movement results from a set of actions applied to the simulated object. The displacements and deformations of the simulated object are related to the actions by the laws of physics.

We can thus characterize a real physical object, or a simulated physical object, by a dipole combining the two dual variables: the extensive ones, positions, and the intensive ones, forces (Figure 1).

Since 1978 the ACROE team has carried out expert evaluations and implementations to design and to build a complete modeller-simulator for physical objects: the Cordis-Anima system. ${ }^{4-12}$

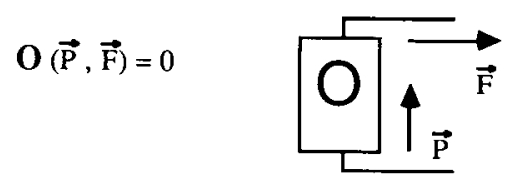

Figure 1. Characterization of a physical object 
These analyses have led to the definition of the basic functions for a modeller-simulator derived from physical modelling. It must enable operator gestural control. The modellization and simulation processes must be real-time oriented.

The two major constraints are:

1. Modularity: the operator must be able to build all kinds of objects and scenes, with various non-linear interactions and discontinuities.

2. Experimentability: the relationship between the model to simulate and the simulation algorithms themselves must run as quickly as possible in order to achieve numerous simulation tests to adjust the models and, at the same time, to produce valid simulations.

These constraints cannot be achieved by any kind of models. We have conducted a consistent analysis of the model-types proposed by physics and the engineering sciences, to extract a principle adapted to this modeller-simulator approach. This led us to the choice of the continuous lumped constant model.

When the objects are too complex or too little known, prior structural discretization is carried out, thus breaking them down into components, each being described by differential equation systems relating the two dual variables force and position (or speed). In fact, the constant localization principle introduces the notion of 'component', and it can be regarded as a language, i.e. as a representation that gives rise to object creation by assembling components. Furthermore, natural phenomena, such as border phenomena (collisions and contacts) or material transformations (agglutination, plasticity), are extremely non-linear. These cannot be represented in an equational form and require state sequencing representation. The latter can be achieved by this 'components formalism', in which some components are linear and others non-linear.

With this model type a system of interacting objects is represented by a discrete network (Figure 2). The nodes represent the material components (point masses), and the links represent interactions between these components.

A simulation algorithm corresponds to each element (nodes and links). It computes a specific dynamic function. To each link, we associate a quadrupole which computes the forces as a function of the positions of the two connected masses. The calculation is made according to a specific interaction law:

$$
\mathbf{F}_{1}=-\mathbf{F}_{2}=\boldsymbol{\Phi}\left(\mathbf{P}_{1}, \mathbf{P}_{2}\right)
$$

When we have obtained these forces, for each mass, a dipole computes the new position according to the Newtonian laws:


Figure 2. The general lumped constant model 


$$
\sum \mathbf{F}=M \Gamma=M \frac{\mathrm{d}^{2} \mathbf{P}}{\mathrm{d} t^{2}}
$$

This model type is mainly oriented towards the explicit representation of the interactions which are generated by physical objects. For example, it is adapted to predict the influence of the environment upon the motions effectively produced by a vehicle.

\section{Models for the environment and for the robot}

In our problems the physical objects to be considered are the ground or obstacles to be crossed over, the obstacles to avoid, and the functional components of the vehicle.

The ground data are more often available as CAD models with either additional information or hypotheses about the sort of ground the vehicle has to move over (it could be sand, mud, stones, etc.). This means that the geometrical models of the ground have first to be converted into appropriate representations. We must achieve a physical representation for the ground in the form of a set of point masses with associated non-penetration areas. We have called these components (mass + non-penetration area) the 'balls', they allow the representation of pieces of matter in the form of 'agglomerates'. ${ }^{10,11}$

CAD models only convey information about the ground topology. But we need, for the physical simulation, a texture model for the robot-ground interactions. This involves additional information about the kind of terrain, and, in fact, about the kind of interactions the vehicle is liable to encounter.

We have developed a set of interaction types, such as elastic and viscous interactions, dry friction or grip-like interaction (notching phenomena), and intermolecular links. The commutation between different interactions laws allows us to represent complex composed interactions such as interactions composed of grip and viscous laws.

In addition, physical models of the vehicle have to exist in the form of a library of various complexity level models which is elaborated by an expert designer using the Cordis-Anima modeller-simulator. In this way, the discrete physical models of several vehicle types such as tracked vehicles, wheeled vehicles and vehicles with several articulations have been constructed and tested with the modeller-simulator (see the 'Examples and Simulations' section).

\section{Operator control and gestural perception}

In a dynamic system, wherever the signals (force/position couples for instance) are detected, these signals are dependent on the global state of the object. Suppose we model an object by means of a set of masses, spring-damper and non-linear interactions, and we put this object in interaction with an environment also represented by a discrete network. Finally, suppose we run the simulation of the whole system. In this instance the simulated system can be represented at each point (the masses for instance) as a dipole, whose impedance is that of the whole system yielded to this point. If this point is chosen as a manipulation point, the operator can have physical information about the global physical behaviour of the whole system. This means that if the object is jammed, the operator will sense the jammings 
and their global magnitude. In a complementary way, visualization can localize the jammed points.

Thus the operator can visually and 'energetically' apply an efficient control of the object's motions. This 'external control' provides natural and optimum driving conditions.

The entire network defined at the required complexity level is made up of (Figure 3)

(a) the robot model: it is composed of the models of all the functional components (locomotion system, general morphology, inertia distribution, etc.)

(b) the ground model: it is an approximation of the terrain topology: hollows, hills, rocks, etc.

(c) an interaction model: it is a physical model of the interactions between the vehicle and the ground. This model depends on the kind of ground (mud, sand, hard ground, stones, etc) and on the locomotion means (wheels, tracks, legs, etc.)

\section{INTEGRATION OF GEOMETRICAL KNOWLEDGE AND PLANNING STEP INSTRUCTIONS}

We have therefore adopted the idea of inserting a physical simulator into a motion planning robotic line. This is to test the consistency of produced solutions from a dynamical point of view. Consequently, we have developed some concepts and their associated tools to design and to implement an interface between the different information levels; that of the robotic line, i.e. logical and geometrical models with symbolic representation of the action, and that of the physical simulation: physical structures, evolution of dynamical systems.

\section{Obstacles avoidance by physical modelling: generalized obstacles}

Classically, a natural terrain is composed of two categories of obstacles:

(i) avoidable-and more often, to avoid-obstacles, for which an avoidance strategy is applied

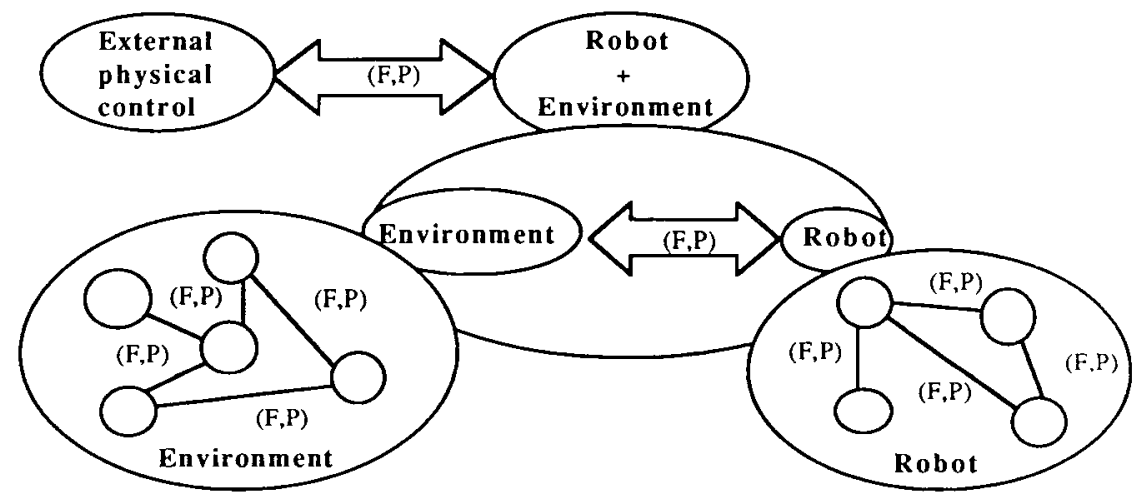

Figure 3. Robot-environment model and operator control 
(ii) unavoidable obstacles, that is to say those where contacts are unavoidable, which are generally not considered, for example the ground. They are of prime importance, particularly for locomotion in a natural environment. We have to reintroduce the effects produced by those obstacles on the motion within the physical simulation.

We have defined the notion of 'generalized obstacles' that embraces in the same notion the two categories. The idea of an obstacle itself is therefore broadened by this notion: the obstacle, that is a nuisance to avoid, becomes an object that can guide motions.

To do this, we transform the obstacle avoidance problem (which is a complex geometrical problem) into a problem of using obstacles as guides for motions. The latter is easier to solve when choosing physical models. We will thus have only one generic problem to solve, which is the physical interaction between physical moving objects.

In order to fully grasp the generalized obstacle's function, let us consider the way the human being, and in fact every living being, deals with obstacle avoidance. We can think about the following anticipation metaphor: everything happens as though the danger processing organ sees objects bigger than they really are. They seem surrounded by a survival area built from the experience of real collisions. We replace, by learning, the visible physical outline of objects by a virtual one which characterizes the safety distance to maintain between the objects and our body. This virtual envelope is a materialized model of anticipation reflexes.

Similarly we will replace every obstacle to be avoided within the simulation, by a virtual protective one used as an anticipator and as a guide for the motions (and of course as a collision avoider with the real obstacle). The distance between real and virtual obstacles depend on path narrowness and hazards.

Notice that those anticipators are adaptive. The virtual obstacles are physical objects with inertia, and whose dynamic behaviour is computed. Thus, they are supposed to move with the vehicle motions, and adapt themselves to the situation (Figure 4).

The definition of generalized obstacle allows the physical simulation to take into account constraints such as safety margins. The real obstacles are localized from the CAD model. Notice that approximate position and shape are sufficient to define the generalized obstacle. Generalized obstacles can either be interactively placed

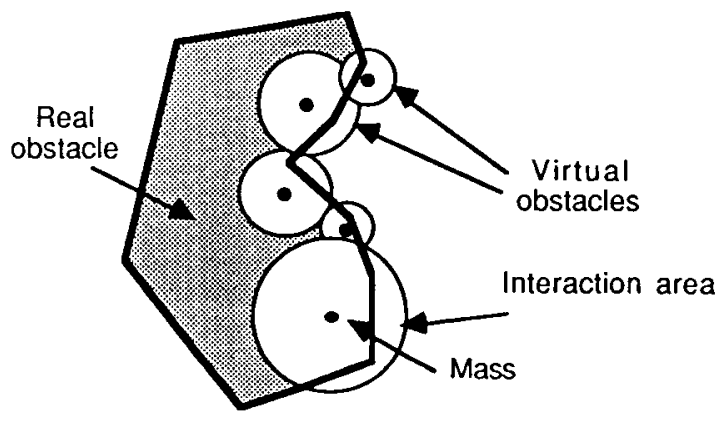

Figure 4. 'Generalized obstacles' 
by an operator or automatically defined according to criteria concerning the real obstacles and safety margins.

The principle can be carried further: a trajectory, such as a channel or polygonal path, could also be directly treated as a physical object, and as such, be materialized by a virtual physical envelope working like the generalized obstacles, and its purpose would be to impose more or less tight trajectory tracking (see key-situation 4 in the 'Examples and Simulations' section and chronogram 1 in Figure 12).

\section{Introducing planning data in a physical world: the 'physical target'}

Targets are usually means to convey and strategic instructions. They can be outputs of planning stages, describing for example subgoal layouts and progression strategies, such as 'step back to be able to pass'. These strategies are related to intelligent behaviour and cannot be fulfilled by physical systems.

The question is then to introduce these non-physical planning data into a physical world. The chosen method is to give a physical model of these non-physical data. Like any physical model, this one has two correlated components: a physical object and physical interactions between this object and the vehicle. This model (object and interactions) has to be designed according to the nature of the planning targets (for example, successive target-configurations for a robot).

In this way, the notion called 'the physical target' materializes strategic and tactic orders. As they are physical, the physical targets can be movable and bear temporal sequences of orders, like those describing some manoeuvres.

As a physical elementary Cordis-Anima object, the elementary physical target is a point mass, and the elementary interactions are attraction and non-linear viscoelastic interaction. With these elementary components, we can represent a simple attractive goal for the rover (see key-situation 6 in the 'Examples and Simulations' section). We can force the vehicle to attain a determined spatial objective where we have located the point-like physical target, and this with some parameters to adjust such as spatial goal achievement speed and accuracy (Figure 5).

In this way, we obtain the optimal practicable trajectory to attain the goal according to the dynamics of the physical scene (rover in interaction with the ground) and the avoidance of obstacles.

This point-like physical target can be placed by the operator or can be given by the planning process. Several targets can be combined, by specifying a set of weighted interactions between themselves and the rover. This allows complex strategies, such as the balance between the local and the global level in the planning process, to be defined.

Because they are physical, physical targets can move in the same way as the rover, and then they can be used directly to drive the vehicle for manoeuvring or to estimate local modification of the strategy in real time. In such cases, it seems useful to have a gestural control device that enables physical feedback, because a simple 3D mouse cannot give a faithful reproduction of the decisive physical parameters. The gestural force feedback devices developed by the ACROE team ${ }^{7,8}$ allow this kind of gestural control on simulated physical objects. 
a)

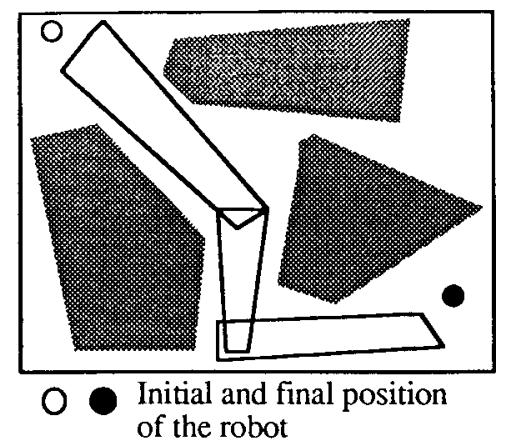

c)

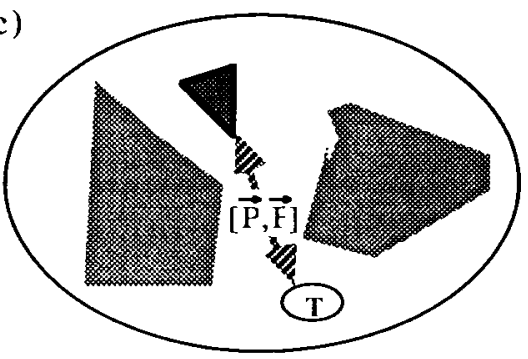

b)

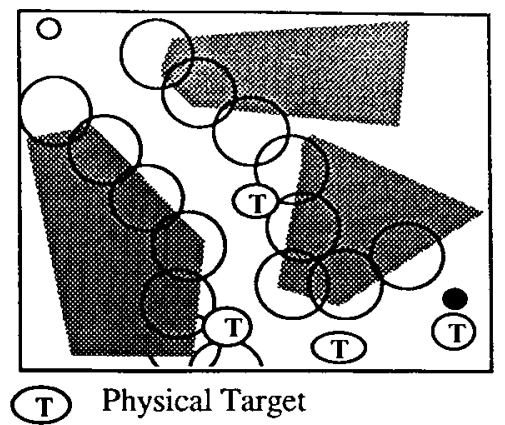

d)

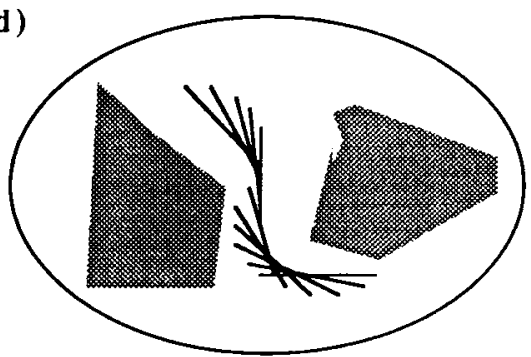

Figure 5. Spatial goal and manoeuvre, geometrical and symbolic reasoning versus physical world solutions: (a) selected safe channel; (b) associated set of generalized obstacles; (c), (d) motion resulting from the combined effect of physical target and vehicle-terrain interaction

\section{Conclusion}

The simulation of dynamics provides assessment of the robustness of trajectory plans coming from the motion planning system, with obstacle avoidance and motion guides. Operator direct control of the physical targets can then be viewed as an ergonomic way of testing some variations on the given solution. It is obvious that the physical simulation can only provide predictions about the possible routes for the vehicle.

The prediction efficiency then depends on the operator's expert evaluations as well as on his ability to exploit the visual and gestural information accessible from the simulation.

The model's flexibility and constructivity, the on-line and real-time simulation (experimentability) make the Cordis-Anima modeller-simulator a complete tool to define efficient task prediction models.

\section{EXAMPLES AND SIMULATIONS}

The Cordis-Anima system, with its real-time simulator, its real-time graphics station and its feedback gestural transducers provides multi-modal information about the simulation performance (Figure 6).

The prediction information produced by the simulation is available in different forms. First, we have talk about the real-time visualization, which is performed by an Evans \& Sutherland PS350 graphics station, and about the operator control by mean of the high performance force feedback devices developed by the ACROE 


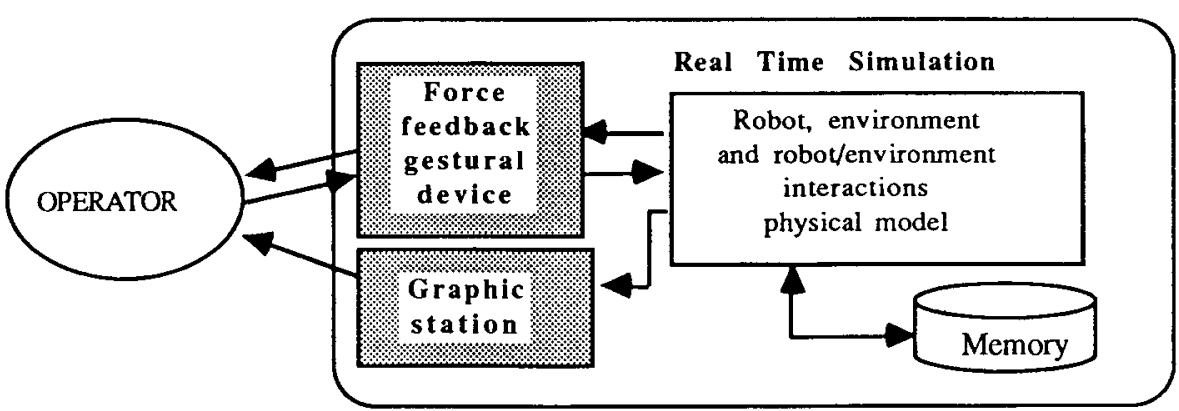

Figure 6. The system: simulation and man-machine communication

team. These represent the available information in line, i.e. during the progress of the simulation. The simulation also carries out the whole scenario of the dynamic evolution of the system. The operator can access the successive states of the simulated system, which is the notion of a dynamic photo. A dynamic photo contains for each time step, the positions, the speeds and the accelerations of each material component, but also the constraints ratio of each interaction link. By analysing these signals we can assess the different variations on motions produced. Assessments are carried out according to criteria such as 'safety', 'required energy', 'accuracy', 'speed' etc. The curves achieved by simulation can then be compared with those elaborated by the robotics experts.

We have experimented with some key-situations using the Cordis-Anima modeller-simulator. These key-situations combine different elements (kind of vehicle, kind of ground, kind of interaction) and the two major concepts defined in this paper: the generalized obstacles and the physical target. The models for the vehicles, for the surfaces, and for the vehicle-ground interactions have been designed and experimented with on the Cordis-Anima workstation.

The implemented models of vehicles are

(a) a 2D articulated vehicle, a 2D tracked articulated vehicle, a flat vehicle with a great number of articulations

(b) a 3D complex articulated rover.

The implemented models of ground are

(a) a frontal view of the terrain composed of rigid and fixed obstacles

(b) a frontal view of the terrain with deformation: moving rigid obstacles, a great number of rigid obstacles to represent stone stacks

(c) a plane view of the terrain with rigid obstacles offering restricted passages

(d) a 3D hilly surface.

The physical interaction laws implemented permit:

(a) to cross over (ground-type) obstacles underneath the vehicle according to non-penetrability and friction parameters

(b) to avoid lateral obstacles according to physical safety margins. 
Key-situation 1: 2D frontal view, 2D articulated vehicle, rigid obstacles underneath Vehicle

The simulation shows a very simplified articulated three-wheel vehicle. The three wheels are represented by point masses with associated non-penetration areas. When the vehicle moves forward, the wheels slide over the ground (Figure 7).

\section{Environment}

This is a $2 \mathrm{D}$ undeformable ground with obstacles to cross over. There are obstacles of different shapes and sizes. The physical model of the ground is a set of different sized 'balls'.

\section{Vehicle-ground interaction}

The interaction between the wheels and the ground is a visco-elastic interaction with non-penetrability.

\section{Locomotion}

The locomotion of the vehicle is provided by a fixed physical target working like a winch.

\section{Key-situation 2: 2D frontal view, 2D tracked articulated vehicle, rigid obstacles underneath}

\section{Vehicle}

The caterpillar track is composed of several point masses connected by springs and dampers in a chaplet form. This string of masses is braced around an articulated body whose structure is the same as above (Figure 8(a)).

The simulation shows the difference with the previous example in real time, particularily the capacity of the track to filter obstacles (Figure 8(b)).

\section{Environment}

Two-dimensional hilly ground (obtained as above) strewn with several point masses. The whole scene looks like a trammel. It creates grip phenomena with the tracked vehicle.

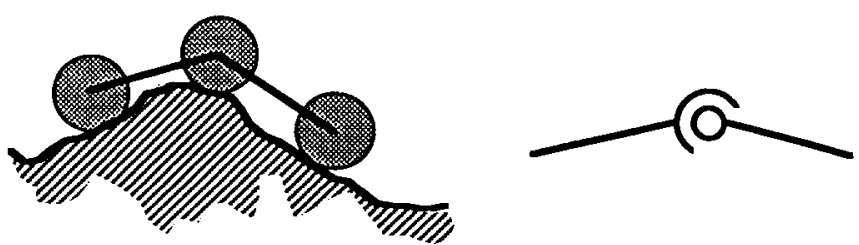

Figure 7. Articulated vehicle getting over rigid obstacles 


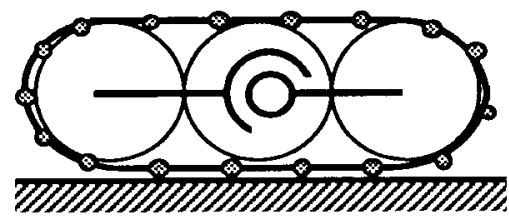

(a)

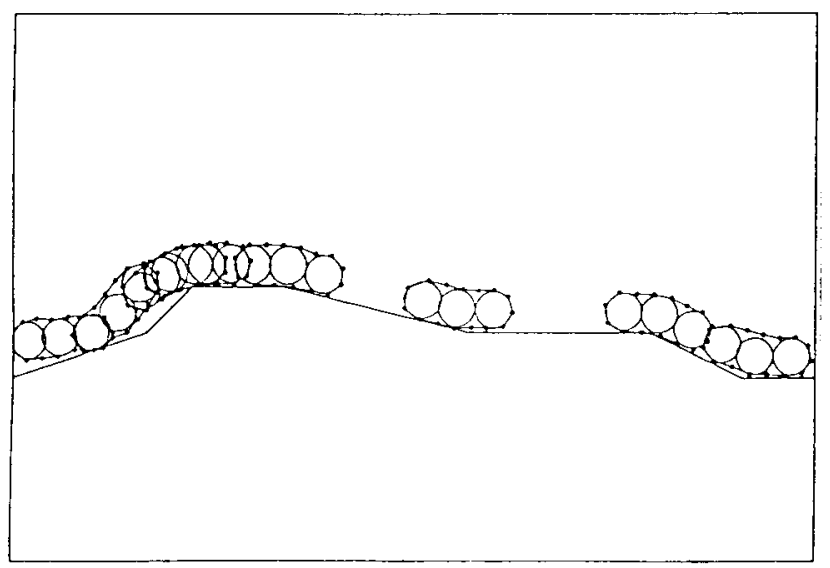

(b)

Figure 8. Tracked vehicle (a), getting over rigid obstacles (b)

The vehicle-ground interaction is similar that in the previous example, but it is applied between each mass fixed on the ground and each mass of the caterpillar track. The locomotion is as above.

\section{Key-situation 3: 2D frontal view, 2D articulated vehicle, deformable obstacles underneath}

The vehicle is the same as in key-situation 1 . The ground is composed of several rigid obstacles that can move. A sand-like ground simulation is obtained when each rigid obstacle is a small element physically linked with others by a physical cohesion law. They can thus constitute stacks and agglomerates. When the vehicle crosses over the stacks, they give way beneath the weight of the vehicle (see Plates 1-3).

Key-situation 4: 2D plane view, flat train-like vehicle, obstacle avoidance and fixed physical target

\section{Vehicle}

It is composed of low deformable elements organized and articulated like a train. This model is a first approach to a robot with many degrees of freedom such as those used in nuclear installations (Plate 4).

\section{Environment}

It contains real obstacles to avoid (yellow polygons in Plate 4) embedded in added virtual obstacles which implement the notion of generalized obstacles (blue spheres in Plate 4). We suppose that a practicable trajectory exists between the real obstacles. This trajectory is materialized by the protective generalized obstacles which are less or more penetrable. 


\section{Vehicle-ground interaction}

In this situation, there is no contact between the vehicle and the real ground. The vehicle only interacts with the virtual surrounding obstacles. The interaction between the two is a viscoelastic non-penetration area with the presence of nonlinearities (contacts are not permanent).

\section{Locomotion}

The locomotion is obtained in the same way as in previous tests, i.e. with a fixed winch-target. But in this case, unlike the other examples, the position of the target is determinant. Here the target is experimentally placed after several attempts (as the simulation runs in real time, attempts take only a few seconds) until it allows the vehicle to edge its way into the restricted passage.

\section{Key-situation 5: 3D terrain with obstacles, 3D complex Martian rover, non- penetrability and friction interaction}

\section{Vehicle}

The rover is a complex 3D articulated vehicle with many degrees of freedom (see Figure 9). The vehicle has six independent cone shaped wheels composed of point masses. The axes are assembled onto an articulated subframe. This vehicle is able to cross over fairly high obstacles. During the obstacle clearing, the subframe articulates itself and, in this way, adapts itself to the shape of the ground (see Figure 10).

\section{Environment}

Level ground with some obstacles to cross over. The obstacles are discretized by non-penetrable different sized spheres ('balls') according to the terrain spatial bandwidth.

\section{Vehicle-ground interaction}

There are two kinds of interaction between the masses composing the wheels, and the ground or the obstacles. The first one is the classic non-penetration law which gives rigid object properties to the obstacles. The second is needed to obtain slide-free rolling motions for the wheels, unlike in key-situation 2 which used a

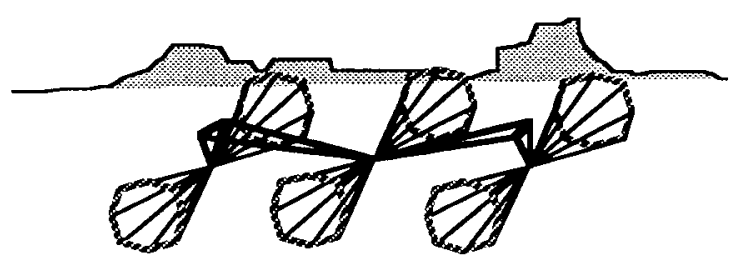

Figure 9. Three-dimensional Martian rover 

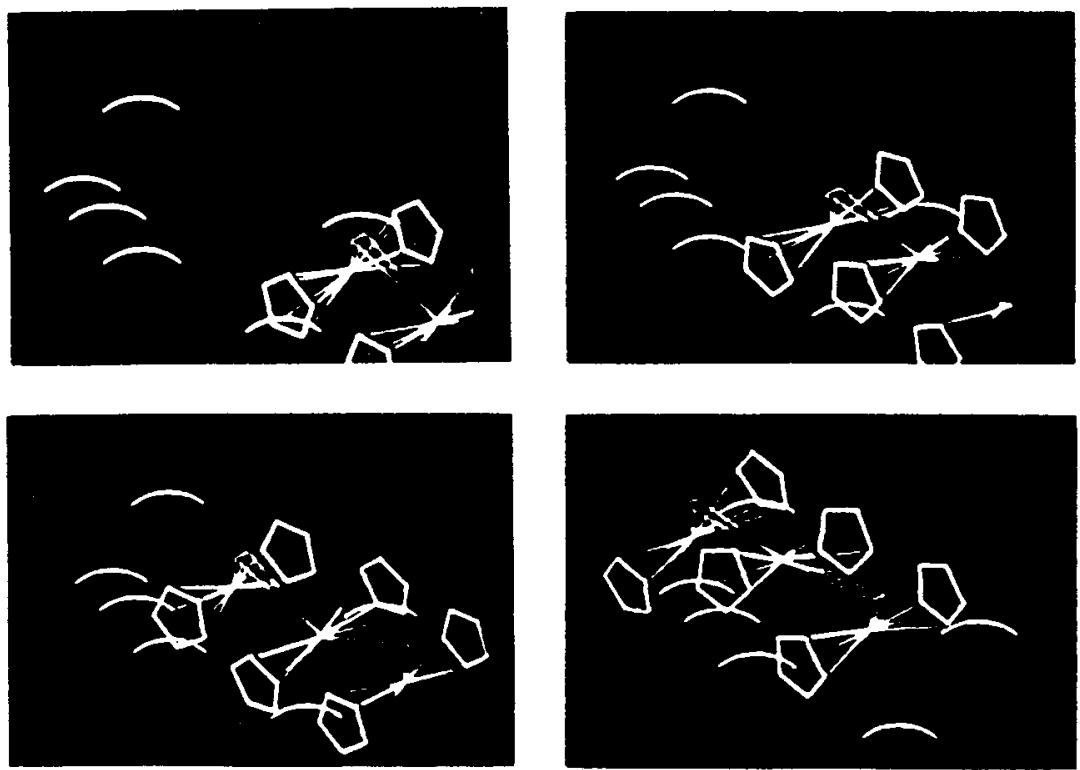

Figure 10. The 3D Martian rover getting over rigid obstacles

trammel-type law. Here we define sliding friction forces that drive the wheels. These forces are proportional to the tangential speed.

In our case, the tangential speed is obtained by projecting the relative speed of the wheel point and the spherical obstacle onto the tangent plane situated at the contact point (see Figure 11).

\section{Locomotion}

In this simulation the 'Martian rover' is still winched by a fixed physical target.

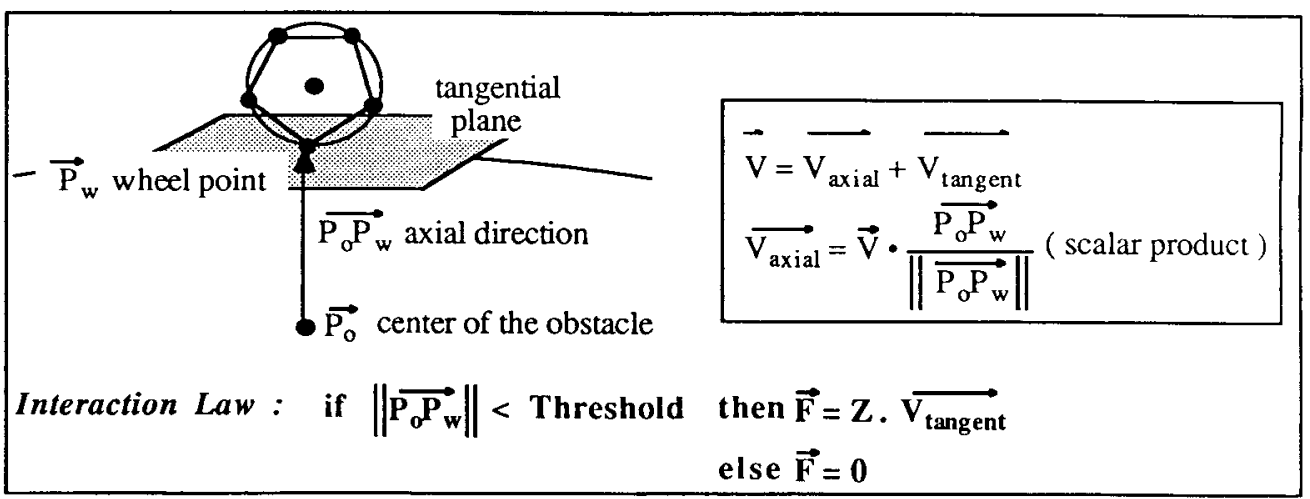

Figure 11. Sliding friction force 
Key-situation 6: moving physical target, operator force feedback control, obstacle avoidance and on-line manoeuvre

The models for the vehicle, the environment and the vehicle-ground interactions are the same as in key-situation 3. However, the practicable channel is more winding and cramped than in previous cases. It does not seem possible to find an optimal path for the vehicle with the use of fixed targets. We have thus used a more adaptive control.

Here the locomotion is provided by a target interactively moved by the operator using a gestural device. The better case is when this device is a force feedback device. ${ }^{8}$ The operator can thus more easily tow the vehicle through the winding passage. Thanks to the feedback forces, the operator feels the possible jams of the vehicle and instantaneously adapts his control.

In this example, we implement both the notion of materialization of a progression strategy and the force feedback motion control for the vehicle by means of a physical target (Figure 12).

\section{CONCLUSION}

We have used lumped constant physical models to make effective motion predictions for robotic vehicles with many degrees of freedom moving in natural environments.
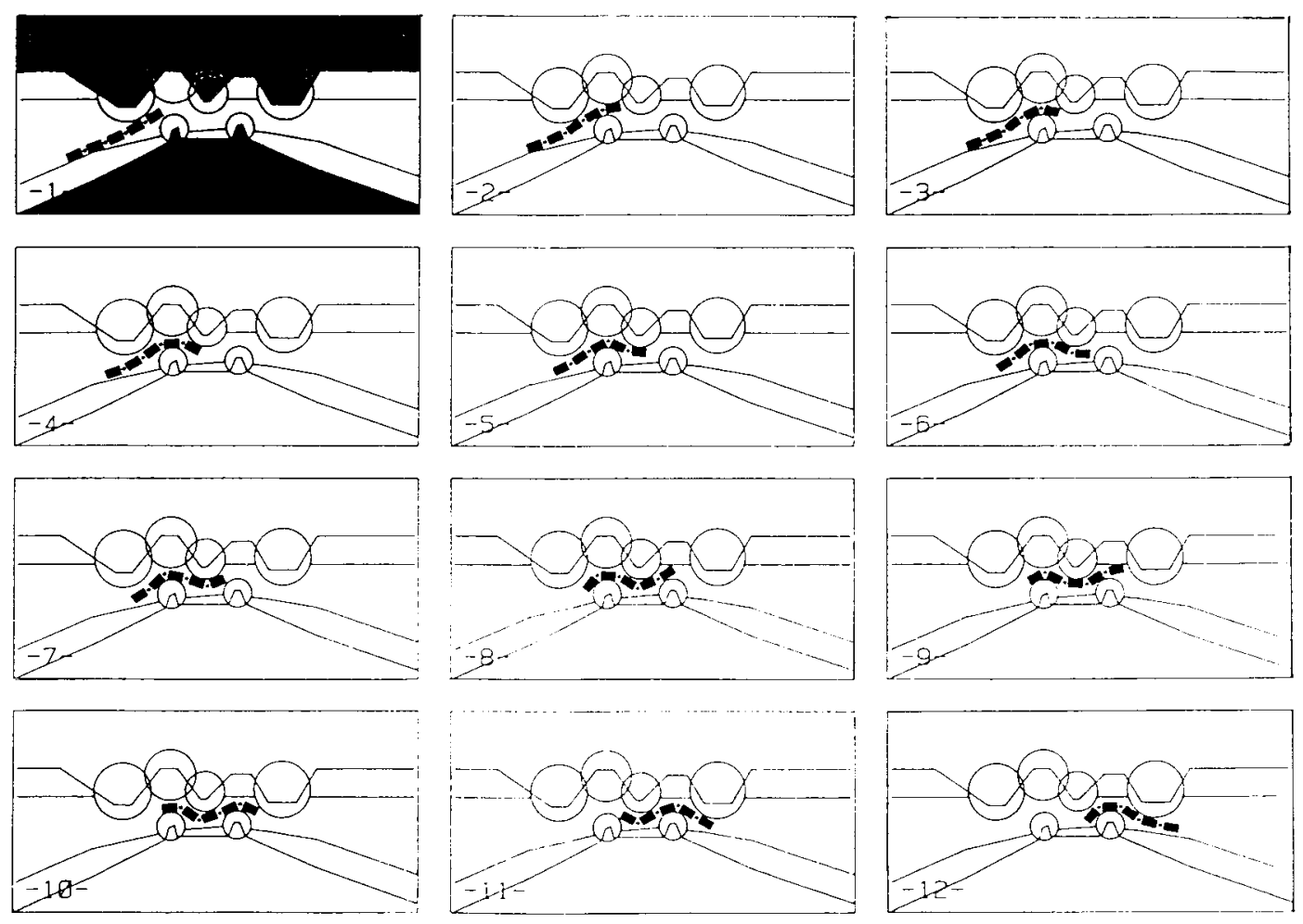

Figure 12. Chronogram, complex motion control for a flat articulated system. Real obstacles are represented by the grey tint polygons on the first picture 
Beyond the inherent difficulties in the design of the models themselves, we have tackled the problems of representation within the physical simulation of symbolic and strategic orders needed for motion control. The Cordis-Anima modeller-simulator for physical objects, initially devoted to animated image synthesis and man-machine communication problems has allowed us to model and to simulate different robotic key-situations.

The concepts of generalized obstacles and of physical target constitute effective solutions to the problem of integrating symbolic information and, beyond this, to the general problem of controlling motions when working with physical models.

Our future work has three axes. First we will aim at making the models of the vehicles more realistic by increasing the level of detail and by modelling actuators and sensors. This goes along with a research work on physical target roles. For example, they can work as a servo-control, by applying a particular command law to some vehicle control points. We have also to define some methods to represent realistic terrain surfaces, i.e. to be able to convert the numerical and CAD models (e.g. face-edge-vertex representations) from real measurement of the grounds into physical models.

This work is related to physics, engineering, automation and programming sciences; it can be viewed as a first step towards a 'physical programming' of robotic systems.

\section{ACKNOWLEDGEMENT}

The research carried out at the ACROE Laboratory is supported by the French Ministère de la Culture (Direction de la Musique et de la Danse). The research work presented here was also supported by the CNES RISP committee (French Spatial Agency).

\section{REFERENCES}

1. J. Barraquant, and J. C. Latombe, 'On non honolomic mobile robots and optimal maneuvring', Revue d'intelligence artificielle, 3, (2), 77-103 (1989).

2. T. Fraichard, C. Laugier and G. Lievin, 'Robot motion planning: the case of non-holonomic mobiles in a dynamic world', IROS'90, Tsuchiura, July 1990.

3. J. Barraquant, B. Langlois and J. C. Latombe, 'Robot motion planning with many degrees of freedom and dynamic constraints', IEEE Int. Conf. on Robotics and Automation, 1989.

4. J. L. Florens, 'Coupleur gestuel interactif pour la commande et le contrôle de sons synthétisés en temps réel', Thèse Doctorat Electronique, INP, Grenoble, 1978.

5. J. L. Florens and C. Cadoz, 'The physical model. Modeling and simulating the instrumental universe', in G. De Poli, A. Piciali and C.Roads (eds), Representation of Musical Signals, MIT Press, Cambridge, Mass, 1991, pp. 227-268.

6. C. Cadoz, 'Synthèse sonore par simulation de mécanismes physiques vibratoires', Thèse INPG, 1979.

7. C. Cadoz, A. Luciani and J. L. Florens, 'Responsive input devices and sound synthesis by simulation of instrumental mechanisms: the Cordis system', Computer Music Journal, 8, (3), (1984). Reprint in Music Machine, MIT Press.

8. C.Cadoz, L. Lisowski and J. L. Florens, 'A modular feedback keyboard design', Computer Music Journal, 14, (2), 47-51 (1990).

9. A. Luciani, 'Physical models in animation: towards a modular and intrumental approach', EUROGRAPHICS Workshop on Animation and Simulation, September 1990.

10. A. Luciani, S. Jimenez, O. Raoult, C. Cadoz and J. L. Florens, 'An unified view of multitude behaviour, flexibility, plasticity and fractures: balls, bubbles and agglomerates', IFIP WG 5.10Modeling in Computer Graphics, Springer Verlag, 1991, pp. 55-74. 
11. S. Jimenez and A. Luciani, 'An unified view of multitude behaviour, flexibility, plasticities and fractures', Rapport de recherche 'Lifia 104-Acroe 89-15', November 1989.

12. S. Jimenez, A. Luciani and $O$. Raoult, 'Simulation et contrôle gestuel en temps réel d'une large variété d'objet physiques...', Journée Informatiques et Graphiques GROPLAN'90. 

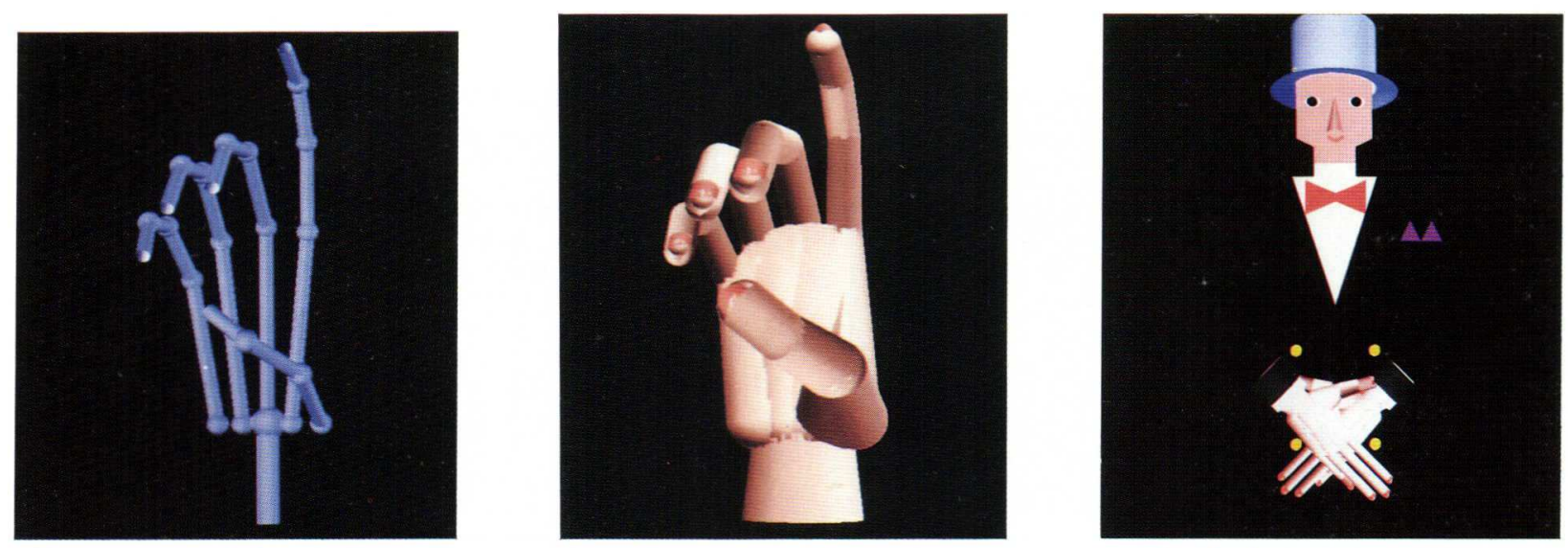

Plate 1 (Lee and Kunii). Skeleton and simplified surfaces of hand

Plate 2 (Lee and Kunii). Signman

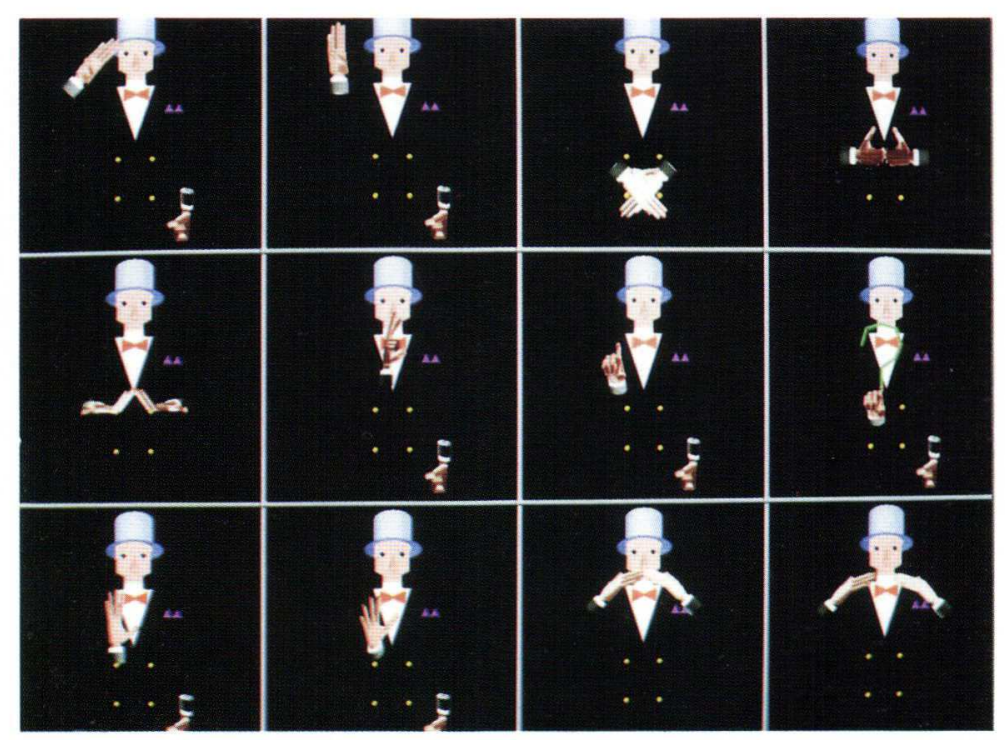

Plate 3 (Lee and Kunii). Common phrases of English by VT

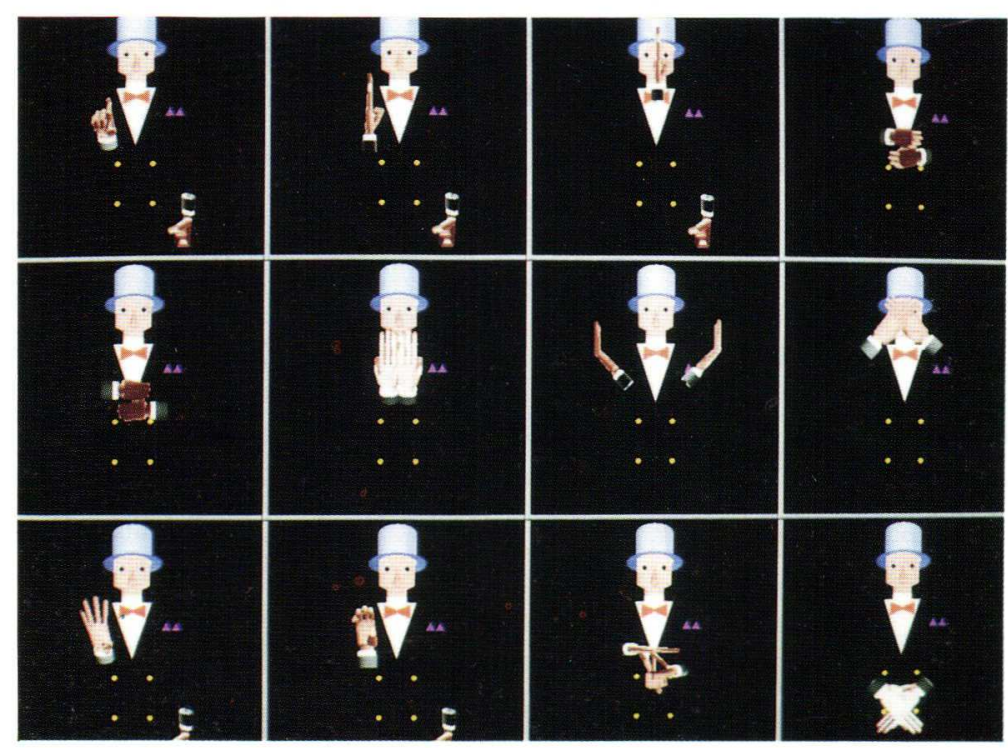

Plate 4 (Lee and Kunii). 'God created heaven and earth' 


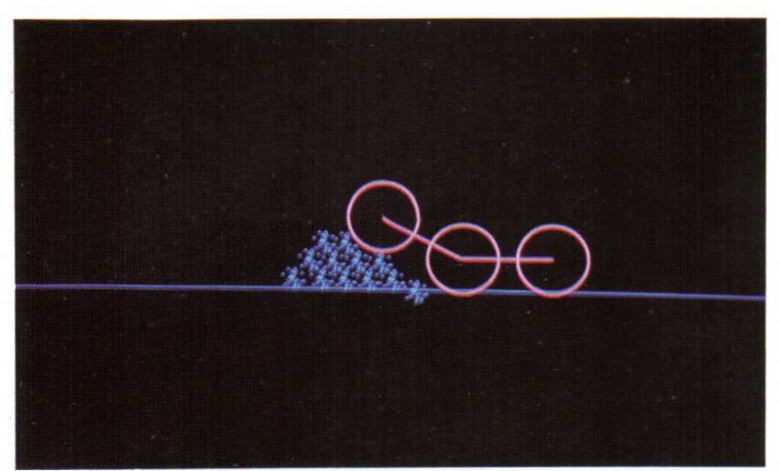

Plate 1 (Jimenez, Luciani and Raoult). Two-dimensional articulated vehicle getting over a deformable obstacle underneath the

$$
\text { vehicle }
$$

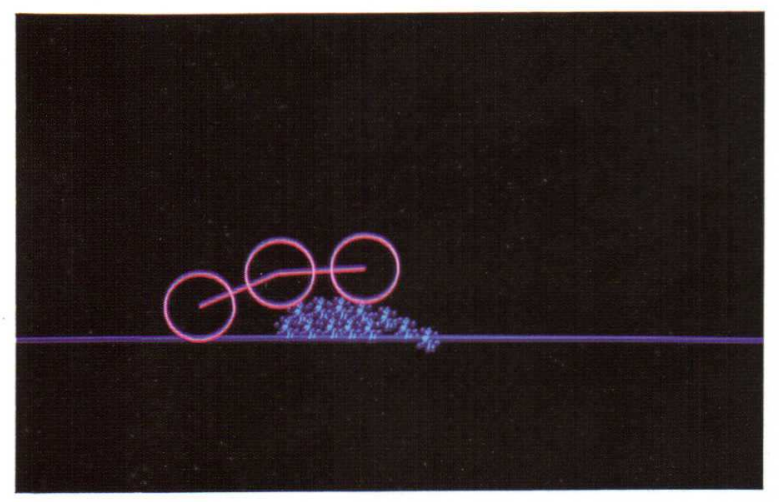

Plate 3 (Jimenez, Luciani and Raoult). Two-dimensional articulated vehicle getting over a deformable obstacle underneath the vehicle



Plate 2 (Jimenez, Luciani and Raoult). Two-dimensional articulated vehicle getting over a deformable obstacle underneath the vehicle

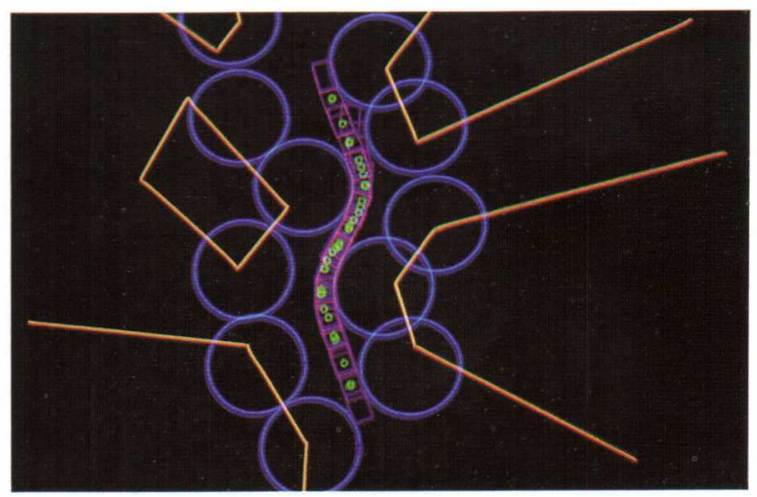

Plate 4 (Jimenez, Luciani and Raoult). Train-like vehicle and obstacle avoidance

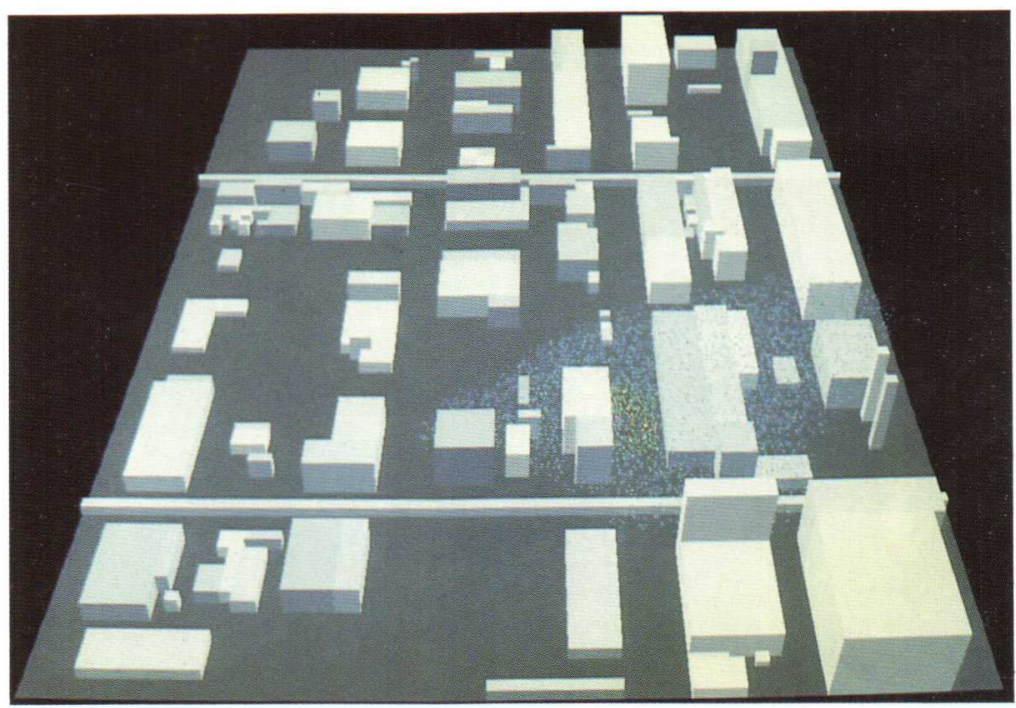

Plate 1 (Kühn and Müller). Pollution distribution over a chemical factory: initial simulation state 


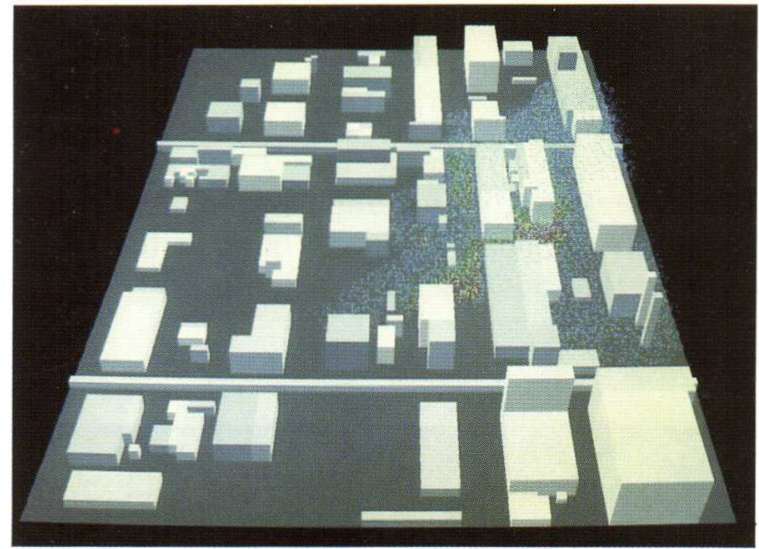

Plate 2 (Kühn and Müller). Pollution distribution over a chemical factory: intermediate simulation state

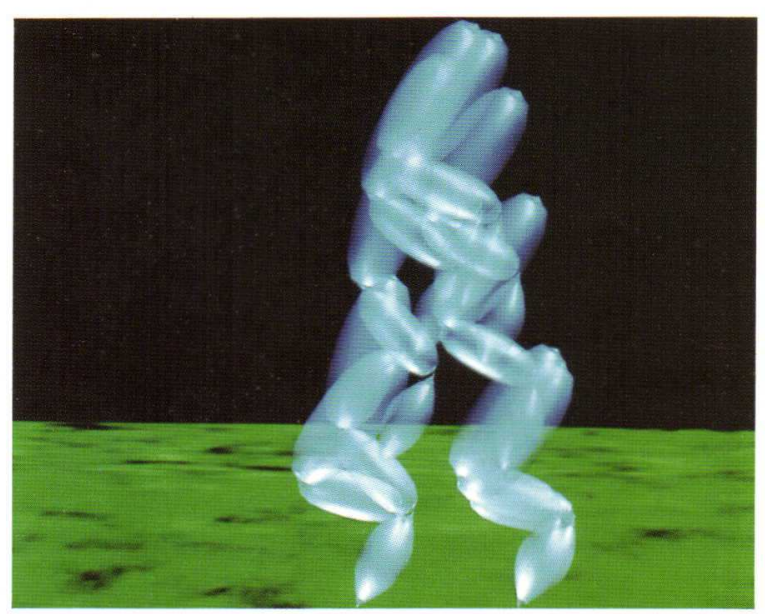

Plate I(a) (Kearney, Hansen and Cremer). Animation sequence for a hopper articulated like a human with links similar to the torso, upper leg and lower leg. A series of frames from a single hop is shown

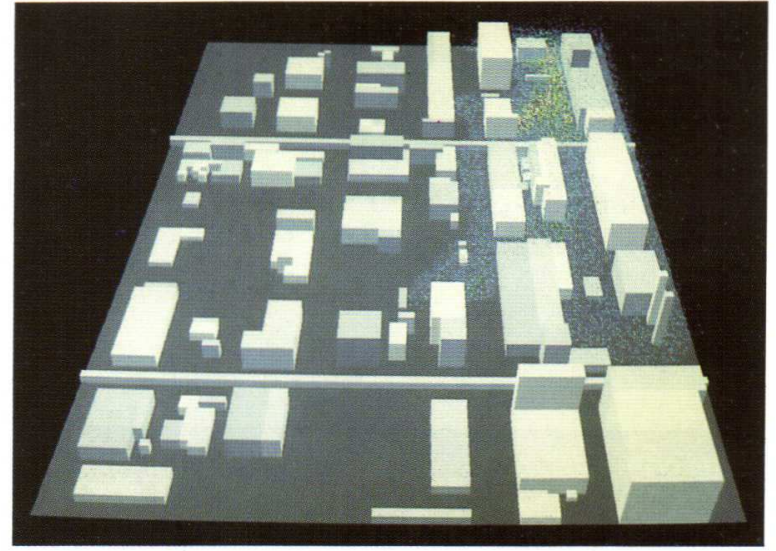

Plate 3 (Kühn and Müller). Pollution distribution over a chemical factory: final simulation state



Plate 1(b) (Kearney, Hansen and Cremer). Animation sequence for a hopper modelled after the one-legged CMU hopper. A series of frames from a single hop is shown



Plate 2 (Kearney, Hansen and Cremer). Simulation results for a human-like hopper in surface contact with the ground during stance. Surface contact enables forward velocity and angular momentum to be decoupled. This permits the hopper to jump vertically with sufficient angular momentum to flip 


\section{Erratum}

In Volume 4, Issue No. 1, the plates associated with the article "Normal vector generation for sampled data using Fourier filtering' by M. E. Goss and I. P. Page (pp. 33-49) were wrongly captioned. The plates for this article are reprinted below with their correct captions.



Plate 1(a) (Goss and Page). Surface generated from Crater Lake elevation data: flat shading

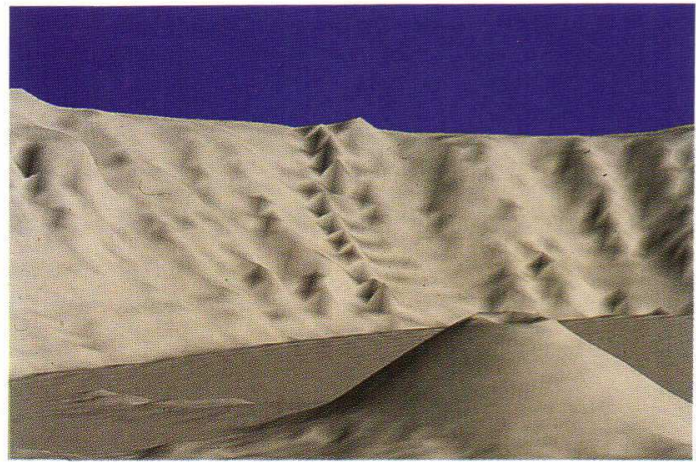

Plate 1(c) (Goss and Page). Surface generated from Crater Lake elevation data: Gouraud shading with DFT normal vectors

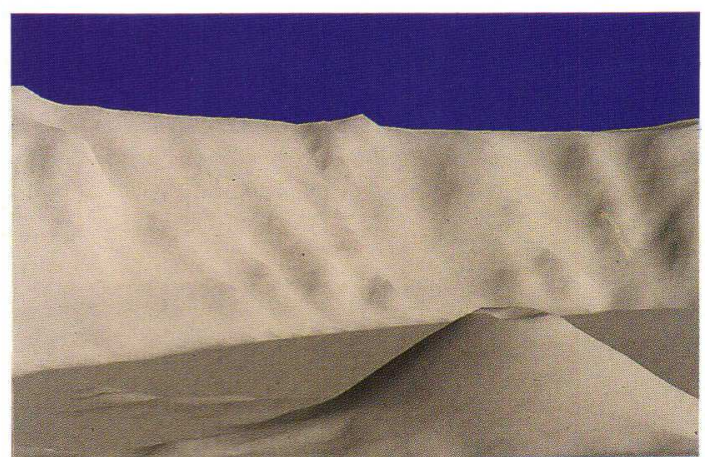

Plate 1(b) (Goss and Page). Surface generated from Crater Lake elevation data: Gouraud shading with average normal vectors

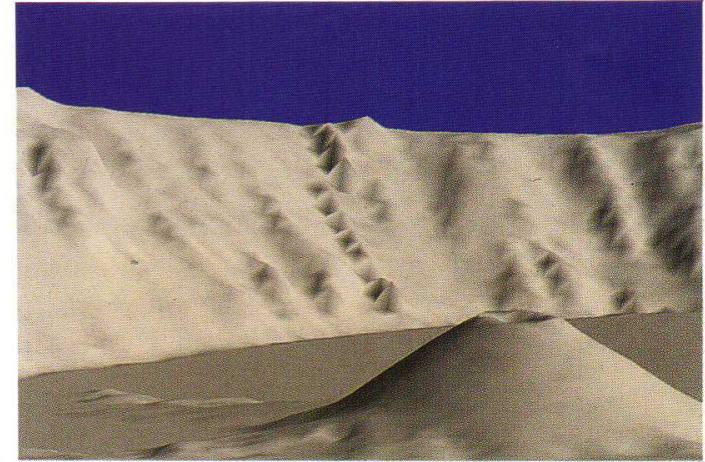

Plate 1(d) (Goss and Page). Surface generated from Crater Lake elevation data: Gouraud shading with DFT normal vectors, lowpass filter

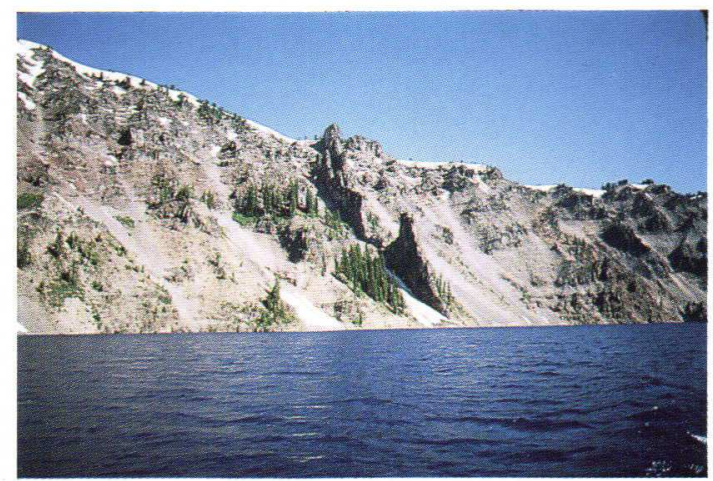

Plate 2 (Goss and Page). Photograph of 'Devil's Backbone' 